\title{
Efeito da idade na forma do fuste de Araucaria angustifolia na região Centro-Sul do Paraná
}

\author{
Ana Paula Marques Martins ${ }^{1 *}$, Sebastião do Amaral Machado ${ }^{1}$, Afonso Figueiredo Filho², Ana Paula Dalla Corte ${ }^{1}$, \\ Maurício Romero Gorenstein ${ }^{3}$
}

${ }^{1}$ Universidade Federal do Paraná, Av. Pref. Lothário Meissner, 632, CEP 80210-170, Curitiba, PR, Brasil

${ }^{2}$ Universidade Estadual do Centro-Oeste, Rodovia PR 153, Km 7, Bairro Riozinho, CEP 84500-000, Irati, PR, Brasil

${ }^{3}$ Universidade Tecnológica Federal do Paraná, Estrada para Boa Esperança, Km 4, CEP 85660-000, Dois Vizinhos, PR, Brasil

*Autor correspondente:

anapaula_marquesm@yahoo.com.br

Termos para indexação:

Crescimento em diâmetro

Forma da árvore

Medição florestal

Index terms:

Diameter increment

Tree form

Forest mensuration

Histórico do artigo:

Recebido em 11/08/2016

Aprovado em 27/05/2017

Publicado em 30/06/2017

doi: 10.4336/2017.pfb.37.90.1329
Resumo - A evolução da forma do tronco de Araucaria angustifolia (Bertol.) Kuntze foi estudada por meio de funções de afilamento e fatores de forma. Os dados utilizados foram obtidos por análise de tronco e por cubagem rigorosa de árvores em pé em povoamentos estabelecidos na região Centro-Sul do Paraná, com uma amostra de 45 árvores. Foram ajustadas quatro funções de afilamento para o conjunto total de dados e para os dados estratificados em classes de idade, sendo construídas as curvas de afilamento para diferentes classes de idade. Foi realizado teste de identidade de modelos para comparar modelos completos com o modelo reduzido. Para avaliar a evolução dos fatores de forma naturais e artificiais, os mesmos foram calculados para o conjunto de dados em diferentes idades, iniciando aos 5 anos. A função de afilamento selecionada foi o polinômio de Schöpfer. Foram observadas diferenças significativas entre o modelo reduzido e os modelos completos, com ligeira vantagem com o uso de equações ajustadas por classes de idade, além de expressiva melhoria na forma do tronco com o aumento da idade, que também foi constatada pela estabilização dos valores de fator de forma aos 22 anos.

\section{Effect of the age on them stem form of Araucaria angustifolia in Central-Southern region of Paraná}

\begin{abstract}
The evolution of the stem form of trees in stands of Araucaria angustifolia (Bertol.) Kuntze was studied by means of taper functions and form factors. The data used were obtained by complete stem analysis and by rigorous scaling of standing trees in stands established in central-southern region of Paraná, with a sample of 45 trees. Four taper functions were adjusted for the total data set and for stratified data by classes of age. Tapering curves were constructed for different age classes. Model identity tests were performed to compare complete and reduced models. To assess the evolution of natural and artificial form factors, they were calculated for different ages, starting at 5 years old. Schöpfer's polynomial was the selected model. There were significant differences among complete and reduced models with a slight advantage in the use of equations adjusted by age classes, as well as a significant improvement in the stem taper with the increase in age, also verified by the stabilization of the values of form factor at 22 years.
\end{abstract}




\section{Introdução}

Araucaria angustifolia (Bertol.) Kuntze, conhecida popularmente como araucária ou pinheiro-do-paraná, é a árvore símbolo da Floresta Ombrófila Mista. Essa formação florestal ocupava grande parte do Paraná antes da sua devastação para implantação de áreas agrícolas, para pecuária e por empresas madeireiras (Maack, 1981).

De acordo com o Anuário da Indústria Brasileira de Árvores (2016), no ano de 2015 havia 11.038 ha de área plantada com araucária em todo o Brasil. Porém, essa área não sofreu grandes mudanças desde 2010 (11.190 ha) o que evidencia a falta de incentivo e a forte pressão legal por parte dos órgãos governamentais que dificultam o cultivo da espécie.

Ainda que a produtividade potencial da araucária seja menor do que se obtém hoje com espécies do gênero Pinus e Eucalyptus, devem-se considerar as possibilidades de uso múltiplo e os benefícios ambientais do cultivo florestal dessa espécie. O desenvolvimento de pesquisas que proporcionem um maior entendimento do crescimento, produção e aproveitamento da araucária pode contribuir para o aumento do interesse de sua utilização em plantios comerciais, possibilitando a retomada de sua competitividade no mercado florestal e assegurando sua sustentabilidade por meio do equilíbrio entre a produção e a conservação.

O mercado demanda cada vez mais variedade e qualidade de produtos florestais. Além disso, as empresas do setor buscam maximizar o aproveitamento de toras e, consequentemente, a renda obtida com seus plantios, investindo na produção de multiprodutos. Nesse contexto, uma importante ferramenta para esse planejamento florestal é o conhecimento de como a forma do fuste das árvores evolui com o avanço da idade (Kohler et al., 2016). Árvores com mesma altura, mas com diferentes formas, produzem produtos diferentes e, consequentemente, tem valor econômico diferente (Sharma \& Parton, 2009).

Dentre os métodos utilizados para expressar a forma do tronco, destacam-se as funções de afilamento e o fator de forma. As equações de afilamento do fuste descrevem o perfil médio das árvores, possibilitando a visualização das diferentes formas geométricas que as porções do fuste assumem no decorrer do tempo (Finger, 1992). O desenvolvimento dessas equações é um pré-requisito básico para estimar volumes de árvores individuais e o rendimento dos produtos florestais (Sharma \& Parton,
2009). Ademais, segundo Li \& Weiskittel (2010), apesar de numerosos modelos de afilamento estarem disponíveis na literatura, é importante desenvolver e ajustar modelos para espécies florestais brasileiras, a fim de incentivar a pesquisa e, consequentemente, o uso das mesmas no mercado florestal nacional e internacional.

O fator de forma é a razão entre o volume cubado da árvore e o volume do cilindro com diâmetro e altura iguais aos da árvore, sendo utilizado como fator de correção para obtenção do volume. A medida que o fator de forma se aproxima de um, mais cilíndrica é a árvore (Finger, 1992).

Além da relevância econômica destas pesquisas, é necessário reforçar a importância de estudos com espécies florestais nativas da flora brasileira, que servem também como forma de incentivo à conservação. Assim, o objetivo desse trabalho foi estudar a evolução do afilamento do tronco no decorrer do crescimento de árvores provenientes de plantios comerciais de $A$. angustifolia localizados na região Centro-Sul do Paraná.

\section{Material e métodos}

\section{Coleta de dados e área de estudo}

Os dados utilizados nesta pesquisa provêm de 45 árvores de Araucaria angustifolia de plantios comerciais em Quedas do Iguaçu, região Centro-Sul do Paraná. De acordo com a classificação climática de Köppen, essa região apresenta clima do tipo Cfa (Instituto Agronômico do Paraná, 2015) e os tipos de solos predominantes na área de estudo são Latossolos e Nitossolos (Embrapa, 2012).

A análise de tronco completa (ANATRO) foi realizada em 30 árvores com 24 anos, sendo utilizados os dados referentes às idades de 6, 12, 18 e 24 anos. Esse povoamento foi implantado em 1990, por semeadura direta, apresentando espaçamento final de $3 \mathrm{~m} \times 2 \mathrm{~m}$ após o "raleio". Nesse plantio foram realizados dois desbastes: o primeiro misto (sistemático e seletivo), aos 18 anos, e o segundo apenas seletivo, aos 22 anos. Para complementação das análises, foram cubadas em pé 15 árvores com 43 anos em um plantio incorporado às áreas de preservação. Esse plantio foi realizado em 1971 com o mesmo método de plantio e espaçamento. Porém, as informações sobre os desbastes não foram disponibilizadas. Dessa forma, o banco de dados total conta com 120 observações provenientes da ANATRO 
e 15 da cubagem rigorosa de árvores em pé. Essas árvores foram selecionadas de acordo com a distribuição diamétrica no povoamento.

Após a derrubada das árvores, foram coletados discos às alturas: 0,$10 ; 0,70$ e 1,30 ; e a cada $2 \mathrm{~m}$ para 15 árvores; e a cada 2,60 m para as outras 15 árvores até o início da copa; a partir do início da copa, o comprimento da seção foi reduzido para $1 \mathrm{~m}$. Após secos e lixados, os anéis de crescimento foram marcados e medidos com o auxílio do equipamento LINTAB 6.0. O crescimento passado da árvore em diâmetro, altura total, volume, assim como o perfil do tronco em qualquer idade foram determinados com o auxílio de planilha eletrônica.

A cubagem rigorosa das 15 árvores foi realizada com o dendrômetro digital Criterion RD 1000. Mediuse a altura a $1,30 \mathrm{~m}$ do solo, assim como o diâmetro nessa posição, com o auxílio de fita métrica; esse ponto foi marcado como referência para as medições com o dendrômetro. Os diâmetros dessas árvores foram medidos nas alturas de 0,$1 ; 0,7 ; 1,30 \mathrm{~m}$, e a cada $2 \mathrm{~m}$ até o final do fuste.

\section{Estimativa dos diâmetros com casca}

Os diâmetros com casca $\left(d_{c d}\right)$ ao longo do tronco para as idades inferiores a 24 anos foram determinados utilizando-se o modelo de Schneider (modificado)
(Equação 1), conforme utilizado por Figueiredo Filho et al. (2015) para a mesma espécie:

$$
\ln d_{c c}=b_{0}+b_{1} \ln d_{s c}+b_{2} \ln \left(h_{i} I\right)+\varepsilon_{i}
$$

Em que: $d_{c c}=$ diâmetro com casca $(\mathrm{cm})$ à altura $h_{i}(\mathrm{~m}) ; d_{s c}$ = diâmetro sem casca $(\mathrm{cm})$ à altura $h_{\mathrm{i}}(\mathrm{m}) ; \mathrm{I}=$ idade (anos); $h_{i}=$ altura $(\mathrm{m})$ onde se encontra o diâmetro $d_{i}(\mathrm{~cm}) ; \mathrm{ln}=$ logaritmo neperiano; $b_{i}=$ coeficientes a serem estimados; $\varepsilon_{i}$. erros aleatórios.

Para aumentar a variabilidade de idades para o ajuste deste modelo, foram incorporadas à base de dados as informações de 38 árvores de A. angustifolia com idades entre 25 e 30 anos, oriundas da FLONA de Três Barras, SC.

\section{Ajuste de funções de afilamento}

Foram ajustados quatro modelos de afilamento de fuste (Tabela 1). A seleção das potências do polinômio de Hradetzky foi feita pelo método Stepwise. As potências inteiras da ordem das dezenas tendem a melhorar o desempenho do modelo na base do tronco da árvore, e as fracionárias a porção superior do fuste. Portanto, foram testadas as seguintes potências: 0,$005 ; 0,09 ; 0,08 ; 0,07$; 0,$06 ; 0,05 ; 0,04 ; 0,03 ; 0,02 ; 0,01 ; 0,9 ; 0,8 ; 0,7 ; 0,6 ; 0,5$; 0,$4 ; 0,3 ; 0,2 ; 0,1 ; 1 ; 2 ; 3 ; 4 ; 5 ; 10 ; 15 ; 20$ e 25 .

Tabela 1. Funções de afilamento testadas para árvores de Araucaria angustifolia em diferentes idades, Quedas do Iguaçu, PR.

\begin{tabular}{cc}
\hline Autor & $\left(\frac{d i}{d a p}\right)^{2}=b_{1}\left(\left(\frac{h i}{h t}\right)-1\right)+b_{2}\left(\left(\frac{h i}{h t}\right)^{2}-1\right)+\varepsilon$ \\
\hline Kozak et al. (1969) modificado & Modelo \\
Schöpfer (1966) & $\frac{d i}{d a p}=b_{0}+b_{1}\left(\frac{h i}{h t}\right)^{2}+b_{2}\left(\frac{h i}{h t}\right)^{2}+b_{3}\left(\frac{h i}{h t}\right)^{3}+b_{4}\left(\frac{h i}{h t}\right)^{4}+b_{5}\left(\frac{h i}{h t}\right)^{5}+\varepsilon$ \\
Hradetzky (1976) & $\frac{d i}{d a p}=b_{0}+b_{1}\left(\frac{h i}{h t}\right)^{p 1}+b_{2}\left(\frac{h i}{h t}\right)^{p 2}+b_{3}\left(\frac{h i}{h t}\right)^{p 3}+\ldots+b_{n}\left(\frac{h i}{h t}\right)^{p n}+\varepsilon$ \\
Garay (1979) & $\frac{d i}{d a p}=b_{1} *\left(1+b_{2} \ln \left(1-b_{3}\left(\frac{h i}{h t}\right)^{b 4}\right)\right)+\varepsilon$
\end{tabular}

Em que: di = diâmetro da seção i $(\mathrm{cm})$; dap = diâmetro a 1,30 $\mathrm{m}$ do solo $(\mathrm{cm})$; hi = altura da seção $\mathrm{i}$; ht = altura total $(\mathrm{m}) ; \mathrm{b} 0, \mathrm{~b} 1, \mathrm{~b} 2, \mathrm{~b} 3$... bn = coeficientes a serem estimados; p1, p2, p3 ... pn = potências a serem selecionadas para compor o polinômio de Hradetzky; $\ln =\operatorname{logaritmo~neperiano.~}$ 
Inicialmente, foram ajustadas as quatro equações para o conjunto de dados referentes às idades selecionadas previamente (6, 12, 18, 24 e 43 anos). Este conjunto foi denominado conjunto total e, após a seleção da equação de melhor desempenho, a mesma foi ajustada novamente para os dados estratificados para as referidas classes de idades.

Os ajustes das funções foram realizados com o auxílio de planilha eletrônica Excel® 2013 e das ferramentas Solver e Action Stat desse mesmo programa.

\section{Seleção das equações de melhor desempenho}

A seleção tanto da equação para estimativa de diâmetro com casca quanto da função de afilamento de melhor desempenho foi realizada baseada nas seguintes estatísticas de ajuste e precisão de modelos: coeficiente de determinação ajustado $\left(\mathrm{R}^{2}{ }_{\mathrm{aj}}\right)$, erro padrão da estimativa em porcentagem $\left(\mathrm{S}_{\mathrm{yx}} \%\right)$ e a análise gráfica dos resíduos.

Tanto o $\mathrm{R}_{\text {aj }}^{2}$ quanto o $\mathrm{S}_{\mathrm{yx}} \%$ foram primeiramente calculados para a variável dependente estimada para cada modelo e posteriormente recalculadas para a variável de interesse de cada equação.

\section{Função de afilamento}

A evolução da forma dos troncos foi avaliada a partir dos gráficos relacionando diâmetro relativo (di/dap) versus altura relativa (hi/ht), construídos com as funções de afilamento selecionadas em cada classe de idade.

Além desta avaliação visual, foi realizada análise estatística, buscando-se detectar diferenças significativas entre as curvas em cada classe de idade. Para tanto, foi empregado o teste de identidade de modelos proposto por Graybill (1976). Esse teste é baseado na diferença entre a soma de quadrados dos resíduos do modelo completo e a soma de quadrados dos resíduos do ajuste do modelo reduzido.

Para se verificar a igualdade entre equações de regressão, testou-se a hipótese de nulidade conforme Graybill (1976).

\section{Fator de forma}

Para a avaliação da evolução do fator de forma ao longo do tempo, foram calculados os fatores de forma natural e artificial de cada árvore para todas as idades, iniciando-se aos 5 anos.

O fator de forma natural $\left(f_{0,1}\right)$ foi calculado tendo como base o diâmetro com casca a $0,1(10 \%)$ da altura total de cada árvore (Equação 2). Para tanto, esses diâmetros a $10 \%$ da altura foram calculados com a interpolação das áreas transversais obtidas na ANATRO.

$$
f_{0,1}=\frac{V o l_{a r v}}{V o l_{c i l}}
$$

Em que: $V_{\text {arrv }}=$ volume total com casca da árvore $\left(\mathrm{m}^{3}\right)$; $V o l_{c i l}=$ volume do cilindro com área transversal referente ao diâmetro a $10 \%$ da altura total da árvore.

$\mathrm{O}$ fator de forma artificial $\left(f_{1,3}\right)$ foi calculado utilizando a equação 2 , sendo que o diâmetro com casca utilizado como referência para o cálculo do volume do cilindro foi a $1,30 \mathrm{~m}$ do solo (DAP).

\section{Resultados}

A equação de Schneider (modificada) (Equação 4) propiciou resultados satisfatórios para a estimativa dos diâmetros com casca ao longo do fuste ( $\mathrm{Syx} \%=2,66 \%$ e $\left.\mathrm{R}^{2} \mathrm{aj}=0,995\right)$, além de apresentar distribuição gráfica dos resíduos com tendenciosidade observada apenas para os diâmetros menores (Figura 1).

$$
\operatorname{Ln} d_{c c}=0,31185+0,94946 \ln d_{s c}-0,0102 \ln \left(h_{i} I\right)
$$

Em que: $\ln =$ logaritmo neperiano. dsc $=$ diâmetro da seção sem casca $(\mathrm{cm})$; hi = altura da seção i; I = idade da seção.

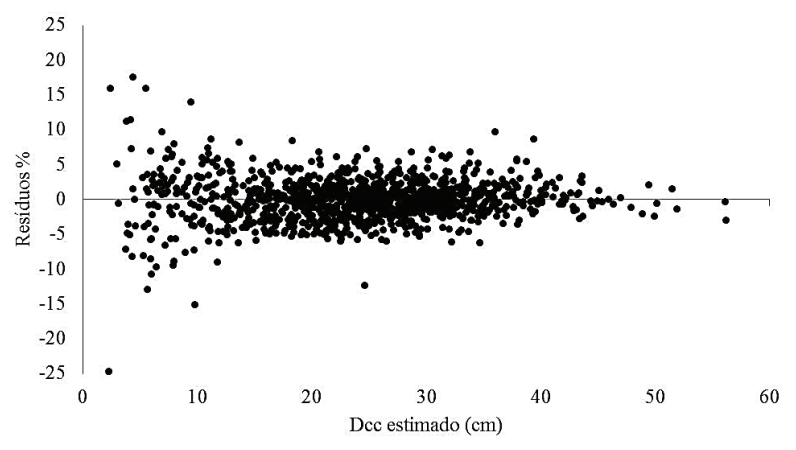

Figura 1. Distribuição gráfica dos resíduos da equação de Schneider (modificada).

Na Tabela 2 observam-se os coeficientes estimados de cada função de afilamento e as respectivas estatísticas de ajuste e precisão dos modelos.

A dispersão gráfica dos resíduos dos ajustes das funções de afilamento pode ser observada na Figura 2.

Tendo em vista que o polinômio de Schöpfer foi selecionado para o conjunto total dos dados, o mesmo também foi ajustado para cada classe de idade separadamente (Tabela 3). 
Tabela 2. Coeficientes estimados, estatísticas dos ajustes e seleção de modelos para o conjunto total dos dados.

\begin{tabular}{ccccccccc}
\hline Modelo & $\mathbf{b}_{\mathbf{0}}$ & $\mathbf{b}_{\mathbf{1}}$ & $\mathbf{b}_{\mathbf{2}}$ & $\mathbf{b}_{\mathbf{3}}$ & $\mathbf{b}_{\mathbf{4}}$ & $\mathbf{b}_{\mathbf{5}}$ & $\mathbf{S}_{\mathbf{y x}} \mathbf{\%}$ & $\mathbf{R}_{\text {aj }}^{\mathbf{2}}$ \\
\hline Kozak et al. (1969) mod. & - & $-1,6567$ & 0,3781 & - & - & - & 11,3 & 0,971 \\
Schöpfer (1966) & 1,1747 & $-2,8091$ & 12,326 & $-28,998$ & 30,638 & $-12,332$ & 9,40 & 0,980 \\
Hradetzky (1976) & 1,1852 & 0,1121 & $-0,5807$ & 0,8525 & 0,4792 & 0,3418 & 9,30 & 0,981 \\
Garay (1979) & 1,2643 & 0,2206 & 0,9894 & 0,2239 & - & - & 10,55 & 0,975 \\
\hline
\end{tabular}

Em que: $b_{0}, b 1, b 2, b_{3} \ldots b n=$ coeficientes da equação, Syx $\%=$ erro padrão da estimativa em porcentagem, $\mathrm{R}^{2} \mathrm{aj}=$ coeficiente de determinação ajustado.
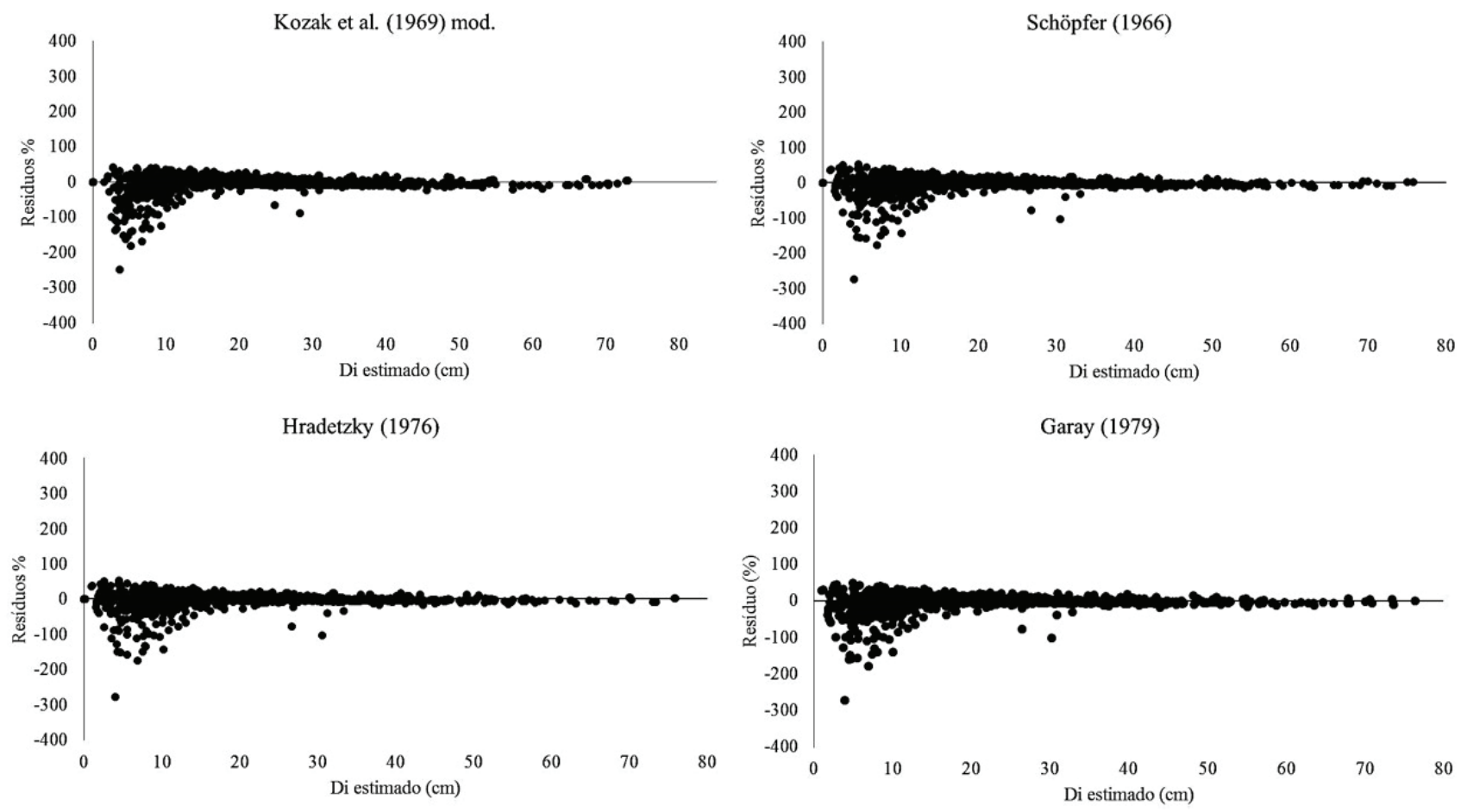

Figura 2. Dispersão dos resíduos das funções de afilamento ajustadas para os diâmetros de seção $i$ (Di) para o conjunto total de dados.

Tabela 3. Coeficientes e estatísticas de ajuste e seleção para o polinômio de Schöpfer ajustado por classes de idade.

\begin{tabular}{ccccccccc}
\hline Idade & $\mathbf{b}_{\mathbf{0}}$ & $\mathbf{b}_{\mathbf{1}}$ & $\mathbf{b}_{\mathbf{2}}$ & $\mathbf{b}_{\mathbf{3}}$ & $\mathbf{b}_{\mathbf{4}}$ & $\mathbf{b}_{\mathbf{5}}$ & $\mathbf{S}_{\mathbf{y x}} \mathbf{\%}$ & $\mathbf{R}_{\text {aj }}$ \\
\hline 6 & 1,2399 & $-2,5202$ & 10,1936 & $-25,921$ & 27,6316 & $-10,6210$ & 14,0 & 0,948 \\
12 & 1,1601 & $-2,5524$ & 10,7018 & $-23,685$ & 22,3707 & $-7,9961$ & 8,2 & 0,980 \\
18 & 1,1689 & $-3,2217$ & 15,5442 & $-36,780$ & 38,3217 & $-15,0317$ & 7,1 & 0,984 \\
24 & 1,1696 & $-3,2382$ & 15,2055 & $-35,398$ & 37,3796 & $-15,1239$ & 6,8 & 0,984 \\
43 & 1,1395 & $-2,7009$ & 11,2782 & $-25,697$ & 27,2039 & $-11,2170$ & 7,8 & 0,974 \\
Geral & 1,1747 & $-2,8091$ & 12,3266 & $-28,998$ & 30,6382 & $-12,3320$ & 9,4 & 0,980 \\
\hline
\end{tabular}

Em que: b0, b1, b2, b3 ... bn = coeficientes da equação, Syx\% = erro padrão da estimativa em porcentagem, $R^{2} a j=$ coeficiente de determinação ajustado. 
06 anos

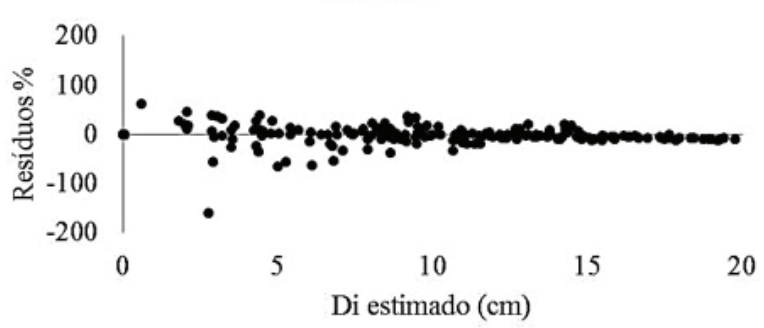

18 anos

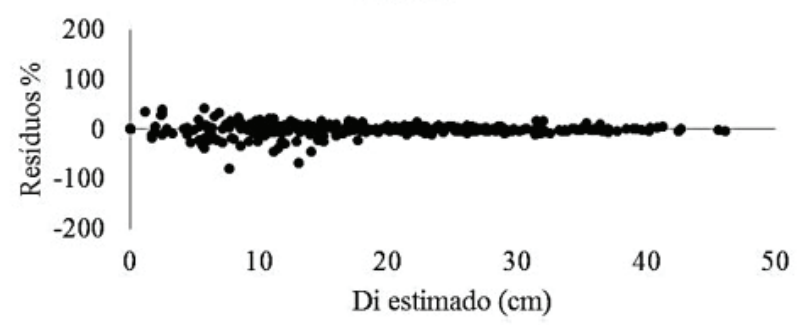

12 anos

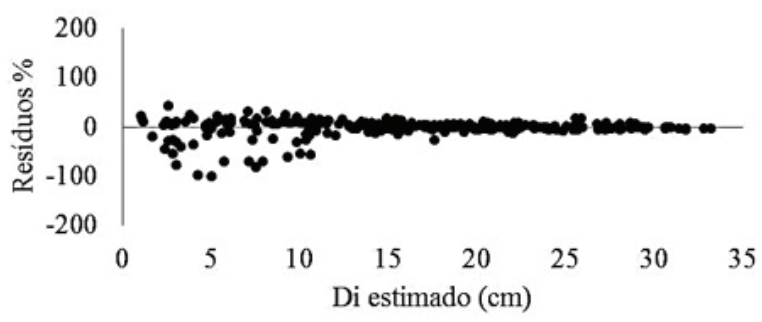

24 anos

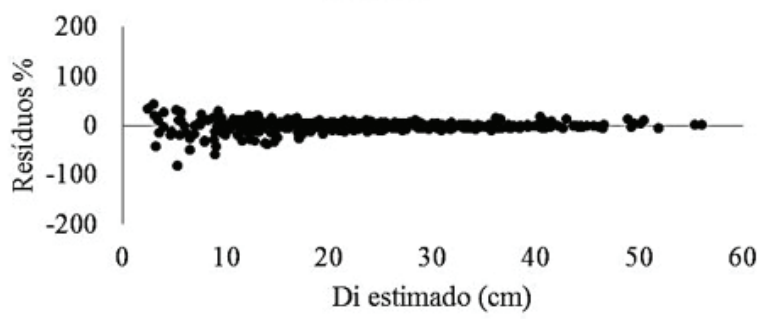

43 anos

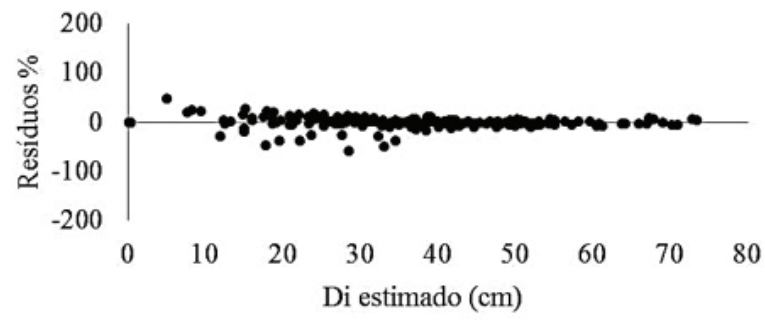

Figura 3. Dispersão dos resíduos para o ajuste do polinômio de Schöpfer para os diâmetros de seção i (Di) para as classes de idade.

Na Figura 3 pode-se observar a dispersão gráfica dos resíduos para as classes de idade. Com o decorrer do tempo, percebe-se uma melhoria na dispersão dos resíduos, porém os maiores erros permanecem nos diâmetros menores.

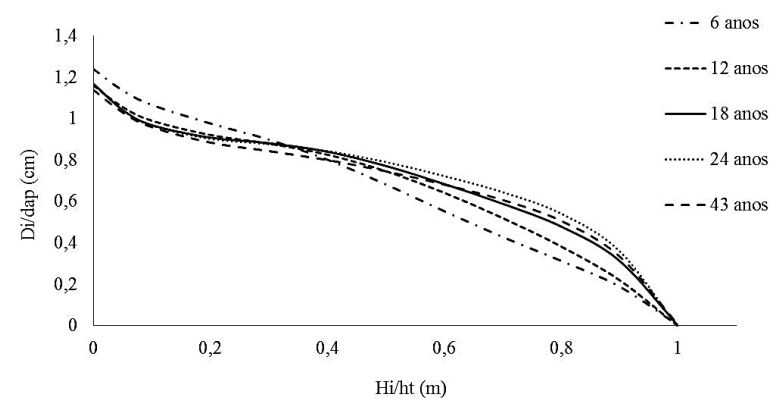

Figura 4. Evolução da forma do fuste de Araucaria angustifolia.
Na Figura 4 observa-se o perfil médio do fuste das árvores de Araucaria angustifolia ajustados com o polinômio de Schöpfer por classes de idade.

Observa-se que nas primeiras classes as curvas são mais afiladas, melhorando o afilamento com o aumento da idade, o que indica uma melhor distribuição do crescimento em diâmetro ao longo do tronco no decorrer do tempo.

De acordo com a ANOVA e com o teste F de Graybill para a identidade dos modelos, houve diferença significativa entre os modelos completo e reduzido referentes às classes de idade analisadas $(6,12,18,24$ e 43 anos), pois o valor de $\mathrm{F}$ calculado foi de $8,3439^{*}$ $(p<0,05)$. Foi necessária, portanto, a estratificação dos dados.

A evolução dos fatores de forma natural e artificial médios calculados por idade podem ser observados na Figura 5. 


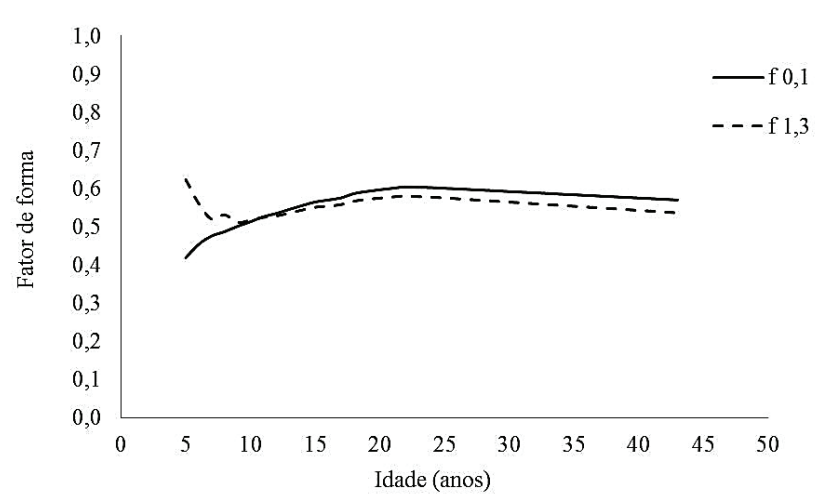

Figura 5. Evolução do fator de forma natural e artificial para Araucaria angustifolia.

Nota-se que o fator de forma natural aumenta com o passar do tempo, indicando melhoria no afilamento das árvores, chegando ao ápice do valor aos 22 anos, quando praticamente se estabiliza, porém tende a sofrer uma leve redução nos anos seguintes.

\section{Discussão}

As equações ajustadas proporcionaram resultados muito semelhantes entre si, com leve vantagem dos polinômios de Hradetzky e de Schöpfer, indicando que qualquer uma das funções poderia ser utilizada para o conjunto total de dados sem que houvesse prejuízo na acurácia das estimativas (Tabela 2). As funções de afilamento apresentaram $\mathrm{R}_{\text {aj }}^{2}>0,97$, o que indica que as funções foram capazes de explicar mais de $97 \%$ da variabilidade dos dados. Além disso, o erro padrão da estimativa alcançado pelas equações foi inferior a $12 \%$.

Para todas as funções, as maiores dispersões de resíduos encontram-se nas estimativas da porção superior do fuste (Figura 2). Isto ocorre devido à maior variabilidade dos dados nas idades mais jovens, assim como devido ao afinamento natural de forma mais abrupta na porção terminal do fuste.

O polinômio de Schöpfer foi selecionado por ser um modelo mais simples e, portanto, proporcionar mais facilidade no ajuste, quando comparado ao polinômio de Hradetzky.

Figueiredo Filho et al. (2015), avaliando o efeito da idade no afilamento do fuste de plantios de $A$. angustifolia com 30 anos de idade, utilizaram o polinômio de Schöpfer para descrever o perfil do tronco para o conjunto total dos dados e obtiveram bons resultados quanto às estatísticas de ajuste e precisão $\left(\mathrm{R}^{2}{ }_{\mathrm{aj}}\right.$
$=0,96$ e $\mathrm{S}_{\mathrm{yx}}=8,85 \%$ ), semelhantes aos obtidos nesta pesquisa. No entanto, Curto et al. (2014), caracterizando a estrutura do povoamento e o desenvolvimento de modelos para quantificação do potencial madeireiro em um povoamento não manejado de $A$. angustifolia, obtiveram os melhores resultados para estimativa dos diâmetros ao longo do fuste com o polinômio de Hradetzky. Entretanto, quando utilizaram as funções de afilamento para estimar o volume total do fuste e por seções do mesmo, o desempenho do polinômio de Schöpfer foi superior.

Costa et al. (2016) com o objetivo de descrever o afilamento do fuste de $A$. angustifolia em um remanescente florestal nativo, considerando a porção que compreende do nível do solo até a inserção da copa, também obtiveram bons resultados com o polinômio de Schöpfer $\left(\mathrm{R}^{2}=0,975\right.$ e Syx $\left.\%=7,5\right)$, o que ressalta o bom desempenho desse polinômio para a espécie.

Diversos autores confirmaram a superioridade dos polinômios de Hradetzky e de Schöpfer na avaliação do afilamento também para algumas espécies de Pinus no Brasil (Araújo et al., 2012; Yoshitani Junior et al., 2012; David et al., 2014).

Loureiro et al. (2012), avaliando equações de afilamento não lineares para um plantio de $A$. angustifolia com 39 anos de idade no município de Rio Negro, PR, concluíram que a equação de Garay foi a que melhor estimou os diâmetros ao longo do fuste $\left(\mathrm{R}^{2} \mathrm{aj}=0,989\right.$ e Syx $=6,93 \%$ ), confirmando a boa performance dessa equação para a espécie.

É possível detectar certa superioridade das equações por classes de idade em relação à equação geral, com significativa diminuição do erro padrão da estimativa para a maioria das classes, exceto para a classe mais jovem (6 anos), sugerindo que a estratificação por idade resulta em melhoria na acurácia das estimativas. Essa tendência de melhoria pode estar relacionada com o aumento da altura e com a melhora da forma com o passar do tempo.

O polinômio de Schöpfer apresentou ajuste um tanto inferior apenas para a classe de idade de 6 anos (Syx $=$ $14 \%$ e $\mathrm{R}^{2} \mathrm{aj}=0,948$ ), devido à maior irregularidade de crescimento em diâmetro em decorrência da falta de competição.

Figueiredo Filho et al. (2014) utilizaram o polinômio de Schöpfer para avaliar a evolução do afilamento de A. angustifolia no município de Laranjeiras do Sul, $\mathrm{PR}$, e obtiveram bons resultados com essa função

Pesq. flor. bras., Colombo, v. 37, n. 90, p. 109-117, abr./jun. 2017 
nas diversas classes de idade, alcançando resultados satisfatórios para a maioria das classes $\left(\mathrm{R}^{2} \mathrm{aj}>0,92 \mathrm{e}\right.$ Syx $<17 \%$ ) para idades superiores a 15 anos. Esses autores também obtiveram piores resultados quanto ao ajuste do polinômio para a classe mais jovem (10 anos), e afirmaram que isto ocorre em razão da maior dispersão dos valores utilizados para o ajuste.

Com o decorrer do tempo, a dispersão dos resíduos tende a apresentar certa melhoria, todavia os maiores erros ainda permanecem nos menores diâmetros (Figura 3). Kohler et al. (2016), estudando a evolução do afilamento de Pinus taeda no Paraná e em Santa Catarina, também constataram que os resíduos se tornavam mais homogêneos com o avanço das classes de idade para os dois grupos de dados amostrados.

Na primeira classe de idade (6 anos) a curva do perfil apresenta, de maneira geral, forma geométrica semelhante a um cone (Figura 4), com ligeira tendência à neilóide na porção até aproximadamente $30 \%$ do fuste. A forma de neilóide da base torna-se cada vez mais acentuada com o decorrer do tempo. Ainda sobre essa classe de idade, nota-se que a partir do ponto correspondente a aproximadamente $30 \%$ da altura total, o perfil torna-se côncavo ao eixo longitudinal.

A classe de idade de 12 anos apresenta comportamento intermediário às classes inferiores e superiores, com o traçado da base acompanhando as classes mais avançadas, e a partir desse ponto apresentando forma cônica. Entretanto, a linha torna-se mais suave em relação à classe de 6 anos, com tendência ao tipo geométrico parabolóide, como o ocorrido nas classes subsequentes.

Para as classes de 18 a 43 anos, na base do tronco as curvas são convexas ao eixo longitudinal, assemelhandose a um sólido geométrico do tipo neilóide, sendo que a partir desse ponto até a base da copa (seção mediana do fuste), o traçado torna-se côncavo em relação ao eixo, assumindo a forma de um sólido geométrico tipo parabolóide até a altura total. Segundo Assmann (1970), este comportamento do traçado na parte mediana até a terminal é normal em coníferas, enquanto que nas folhosas uma parte dessa seção seria parabolóide e a parte terminal até a base da copa seria descrita por um tronco de cone.

Burger et al. (1979) analisaram o desenvolvimento da forma de árvores dominantes de A. angustifolia e ajustaram o polinômio de Schöpfer para obter o perfil das curvas de afilamento nas diferentes idades. Esses autores descreveram que, aos seis anos, a forma das árvores era parabolóide ao longo de todo o tronco, tornando-se neilóide na parte inferior e cada vez mais parabolóide na porção superior do fuste com o avanço da idade, induzindo os autores a concluírem que a forma do fuste melhora com a idade, ou seja, tornase cada vez mais cilíndrica com o decorrer dos anos. Outros autores também investigaram o efeito altamente significativo da idade na melhoria da forma do fuste de árvores. Recentemente, para algumas coníferas como A. angustifolia, este fato foi constatado por Figueiredo Filho et al. (2014, 2015); para P. taeda por Kohler et al. (2016); para P. elliottii por Téo et al. (2013).

$\mathrm{O}$ fator de forma artificial sofre mais oscilações nos primeiros anos de idade do povoamento (Figura 5), apresentando os maiores valores nessas idades iniciais, pois quando a árvore é jovem, o DAP está mais próximo da altura total, tornando o volume do cilindro pequeno em relação ao volume da árvore. Essa relação se inverte à medida que a árvore cresce e a altura do DAP se afasta da altura total. Portanto, o fator de forma artificial não representa diretamente a forma geométrica do tronco da árvore, sendo um fator de redução para o cálculo do volume (Burger et al., 1979).

Além disso, quando o povoamento em questão atinge 9 anos, o fator de forma artificial tem o mesmo comportamento do fator de forma natural, aumentando até alcançar os 22 anos, quando tende a diminuir de forma tênue com o avanço da idade. Essa ligeira diminuição tanto do fator de forma artificial, quanto do natural, pode estar relacionada a desbastes seletivos realizados na área aos 22 anos. Quando o desbaste é realizado, a competição entre as árvores do povoamento diminui, com o favorecimento do crescimento em diâmetro, contribuindo dessa forma para o aumento do afilamento (Larson, 1963).

Observa-se que os fatores de forma natural e artificial apresentam significativa queda nos valores aos 43 anos. Porém, vale ressaltar que esses dados foram coletados em área diferente dos coletados nas demais idades e com outra metodologia. E, portanto, essa diminuição nos valores não significa necessariamente que a forma do fuste da araucária piore em idades mais avançadas.

\section{Conclusão}

As funções de afilamento ajustadas, de maneira geral, descrevem satisfatoriamente o perfil médio das árvores 
estudadas, fornecendo informações concretas quanto à evolução da forma do fuste de Araucaria angustifolia.

Os perfis médios ajustados nas diferentes classes de idade apresentam diferença significativa de acordo com o teste $\mathrm{F}$ de Graybill, indicando que a forma das árvores se altera com o avanço da idade.

O fator de forma natural representa a forma geométrica das árvores, mostrando melhoria da forma do fuste até os 22 anos, quando então observou-se tendência de diminuição, revelando a influência de desbastes realizados no povoamento. $\mathrm{O}$ fator de forma artificial não descreve a forma geométrica das árvores nas idades mais jovens devido à influência do uso do DAP como base para área transversal do cilindro. A partir dos 9 anos do povoamento, esse fator de forma tem comportamento análogo ao fator de forma natural.

\section{Referências}

Araújo, E. J. G. et al. Relações dendrométricas em fragmentos de povoamentos de pinus em Minas Gerais. Pesquisa Florestal Brasileira, v. 32, n. 72, p. 355-366, 2012. DOI: 10.4336/2012. pfb.32.72.355.

Assmann, E. The principles of forestry field study. Oxford: Pergamon Press, 1970. 506 p.

Burger, D. et al. Estudo do desenvolvimento da forma de Araucaria angustifolia com relação à idade. In: ENCONTRO DA INTERNATIONAL UNION FOR FORESTRY RESEARCH ORGANIZATION, 1., 1979, Curitiba. Anais... Curitiba: IUFRO, 1979. p. 320-329.

Costa, E. A. et al. Função de afilamento e sortimentos de madeira para Araucaria angustifolia. Ciência Florestal, v. 26, n. 2, p. 523-533, 2016. DOI: http://dx.doi.org/10.5902/1980509822753.

Curto, R. A. et al. Caracterização da estrutura e desenvolvimento de modelos para quantificação do potencial madeireiro em povoamento não manejado de Araucaria angustifolia. Pesquisa Florestal Brasileira, v. 34, n. 80, p. 303-319, 2014. DOI: 10.4336/2014. pfb.34.80.764.

David, H. C. et al. Critérios de estratificação para o ajuste de funções de afilamento em fustes de pinus. Pesquisa Florestal Brasileira, v. 34, n. 79, p. 197-206, 2014. DOI: 10.4336/2014.pfb.34.79.659.

Embrapa. Mapa simplificado de solos do estado do Paraná. 2012. Disponível em <http://www.escola.agrarias.ufpr.br/arquivospdf/ mapa_solos_pr.pdf>. Acesso em: 28 maio 2015.
Figueiredo Filho, A. et al. Dinâmica do afilamento do tronco e da produção de madeira em plantios de Araucaria angustifolia. Cerne, v. 20, n. 4, p. 595-603, 2014. DOI: 10.1590/01047760201420041386.

Figueiredo Filho, A. et al. Efeito da idade no afilamento e sortimento em povoamentos de Araucaria angustifolia. Floresta e Ambiente, v. 22, n. 1, p. 50-59, 2015. DOI: 10.1590/2179-8087.080114.

Finger, C. A. G. Fundamentos de biometria florestal. Santa Maria, RS: UFSM/CEPEF/FATEC, 1992. 314 p.

Graybill, F. A. Theory and application of the linear model. Belmont: Duxbury Press, 1976. 704 p.

Indústria Brasileira de Árvores. IBÁ: Indústria Brasileira de Árvores. São Paulo: Studio 113, 2016. 100 p. Relatório anual da Indústria Brasileira de Árvores 2016. Disponível em: < http://iba.org/images/ shared/Biblioteca/IBA_RelatorioAnual2016_.pdf $>$. Acesso em: 4 maio 2017.

Instituto Agronômico do Paraná. Cartas climáticas do Paraná: classificação climática segundo Köppen. Londrina, [s.d.]. Disponível em: <http://www.iapar.br/modules/conteudo/conteudo. php? conteudo=597> . Acesso em: 15 jan. 2015.

Kohler, S. V. et al. Evolution of tree stem taper in Pinus taeda stands. Ciência Rural, v. 46, n. 7, p. 1185-1191, 2016. DOI: 10.1590/01038478 cr20140021.

Larson, P. R. Stem form development of forest trees. Forest Science, Monograph 5, n. 4, p. 1-41, 1963. Suplemento.

Li, R. \& Weiskittel, A. R. Comparison of model forms for estimating stem taper and volume in the primary conifer species of the North American Acadian Region. Annals of Forest Science, v. 67, n. 3, p. 302. 2010. DÓI: DOI: 10.1051/forest/2009109.

Loureiro, G. H. et al. Avaliação de equações de afilamento em um plantio de Araucaria angustifolia (Bertol.) Kuntze utilizando o diâmetro como variável dependente. In: CONGRESSO FLORESTAL PARANAENSE, 4., Curitiba, 2012. Anais... [Curitiba]: Malinovski, 2012. Disponível em < http://malinovski.com.br/CongressoFlorestal/ Trabalhos/04-Manejo_Plantadas/MFPlantadas-Artigo-05.pdf $>$. Acesso em: 17 nov. 2015.

Maack, R. Geografia física do Estado do Paraná. Curitiba: José Olympio, 1981. 450 p.

Sharma, M. \& Parton, J. Modeling stand density effects on taper for jack pine and black spruce plantations using dimensional analysis. Forest Science, v. 55, n. 3, p. 268-282, 2009.

Téo, S. J. et al. Modelos de afilamento para Pinus elliottii em diferentes idades, na região de Caçador, SC. Floresta, v. 43, n. 3, p. 439-452, 2013. DOI: 10.5380/rf.v43i3.30320.

Yoshitani Junior, M. et al. Funções de afilamento para plantios desbastados de Pinus taeda. Floresta, v. 42, n. 1, p. 169-176, 2012. DOI: $10.5380 / \mathrm{rf} . v 42 \mathrm{i} 1.26315$. 
